\title{
Balancing the benefits and harms of MRI-directed biopsy pathways
}

\author{
Anwar R. Padhani ${ }^{1}$ [ $\cdot$ Masoom A. Haider ${ }^{2}$. Olivier Rouviere ${ }^{3,4,5}$
}

Received: 7 November 2021 / Revised: 30 November 2021 / Accepted: 3 December 2021 / Published online: 1 February 2022

(c) The Author(s), under exclusive licence to European Society of Radiology 2022

\section{Key Points}

- Before a prostate biopsy, the likely benefits and the harms emanating from true and false test MRI results need to be balanced. Prioritizing patients' preferences and their tolerance to potential harms are essential to assess.

- The decision curve analysis method is an analytical framework where the net clinical benefit is plotted against a range of risk thresholds of having important cancers, helping patients and their physicians to decide between cancer averse (important cancers being detected) and biopsy averse (biopsies avoided) strategies.

- The decision curve analysis method showed that the incorporation of clinical risk factors with MRI findings optimizes biopsy outcomes over a range of clinically relevant risk thresholds, compared to other biopsy strategies.

Keywords Prostate cancer · Diagnosis · Multiparametric MRI · MRI-directed biopsy · Systematic biopsy

Prostate cancer diagnoses in the MRI-directed biopsy era are fraught with the need to make choices, in order to strike the appropriate balance between the benefits and harms for patients in their biopsy decisions. Diagnostic and patient impacts of both true and false test results should influence who and how to conduct a biopsy. Combining in all men with suspected cancer, systematic biopsies and targeted biopsy after a positive MRI scan seems like a safe choice, because it maximizes the detection of important cancers. However, the use of systematic biopsies is associated with an increase in the detection of low-grade cancers that may submit patients, at best, to the extra cost and anxiety of active

This comment refers to the article available at https://doi.org/10. 1007/s00330-021-08407-6.

\section{Anwar R. Padhani}

anwar.padhani@stricklandscanner.org.uk

1 Paul Strickland Scanner Centre, Mount Vernon Cancer Centre, Rickmansworth Road, Northwood HA6 2RN, Middlesex, UK

2 Joint Department of Medical Imaging, University Health Network, Sinai Health System and University of Toronto, Toronto, ON, Canada

3 Department of Urinary and Vascular Radiology, Hospices Civils de Lyon, Hôpital Édouard-Herriot, Lyon, France

4 Université de Lyon, Lyon, France

5 Faculté de Médecine Lyon Est, Université Lyon 1, Lyon, France surveillance and, at worst, to overtreatment. In addition, a prostate biopsy is associated with discomfort and morbidity, and it seems desirable to try to avoid it in patients whose risk of important cancers is reasonably low.

The MRI-directed pathway's ability of rule out cancer (its true-negative test result) is commonly emphasized because it is substantial and most robust. Sathianathen et al. in a meta-analysis of 42 studies reported a negative predictive value of $91 \%$ which varied a little between studies [1], and centres with similar demographic mix show higher degrees of agreement [2]. It is, therefore, reasonable to conclude that MRI, when negative, could help avoid unnecessary biopsies. Nonetheless, attention should be paid to the MRI facility, and to standardizing MRI techniques and radiological assessments [3]. In addition, because the negative predictive value of MRI is dependent on prevalence [4], estimating the patient's likely risk should help assess whether or not we can trust negative MRI findings.

What to do with men with MRI positive findings is more difficult for multiple reasons. Overall, the specificity of MRI is intermediate and is highly variable between studies [5] and centres [6], so it seems desirable to be highly selective on who and how to biopsy men with positive results. Here also, estimating the likely patient risk should help since the positive predictive value is also dependent on prevalence [5]. Although the PI-RADS score is a good predictor of the likelihood of the presence of important cancers, success is dependent on the suspicion category. Therefore, the ideal 
diagnostic method for biopsy should not be the same for category PI-RADS 3 or PI-RADS 5 lesions, provided, of course, that radiological assessments are made using the PIRADS recommendations [7].

We must also not forget the patient impact of false results after an MRI assessment. Fortunately, the high NPV means that there are few men harbouring important cancers after negative MRI; therefore, appropriate safety nets are needed to find these men as their cancers emerge during observations. Some men with positive MRI have biopsies that do not have important cancers on targeted cores but are discovered on accompanying systematic cores [8]. Contributing factors include targeting errors and histologic interpretation variability. The need for quality control and quality assurance procedures through the accreditation of team working and certification of the team members is needed to decrease biopsy yields variability [3].

With these complexities in mind, there are multiple ways to optimize the balance of MRI-directed biopsy results, weighing the benefits versus the harms (Fig. 1). Firstly, it is possible to adjust patient selections by incorporating PSA-density values with positive and negative MRI scan results [9], with or without additional clinical factors that informing on the risk of having an important cancer [10, 11]. Secondly, by thresholding on a different PI-RADS assessment category for biopsy decisions (e.g. score 4 instead of 3), or taking the radiological stability into account in men on active surveillance. Thirdly, the biopsy approach for men with a 'positive MRI scan' result may be altered to ensure that the target has been optimally sampled, for example by increasing the number of targeted cores employed. Fourthly, to compensate for possible histologic grade shifts of targeted biopsy cores, by increasing the core numbers, adjusting the histologic evaluations of the targeted cores [12], or employing a higher histologic grade for clinical significance [8].

When employing these methods, it is necessary to judge the equilibrium between the benefits and harms using a common analytical framework. The decision curve analysis method, in which net clinical benefit is plotted against a range of relevant risk thresholds of having cancer, can give insights into the balance [13]. The net benefit quantifies the net true positives or true negatives corrected for the harm of false positives or false negatives, whereas the risk threshold indicates the maximum acceptable risk of missing a clinically important cancer. If a patient or their physician is particularly worried about missing disease (cancer averse) and/or there is no excess risk of biopsy complications, then a low-risk threshold for biopsy would be reasonable. If, by contrast, a patient or their physician is more worried about the potential harms of biopsy (biopsy averse), then a higher risk threshold may be adopted.

Bittencourt et al. [11] retrospectively evaluated the impact of different diagnostic pathways, in a cohort of biopsy-naïve men at high risk for prostate cancer, who came to their reference centre for MRI-ultrasound fusion prostate biopsy. They compared systematic biopsies in all patients, with different

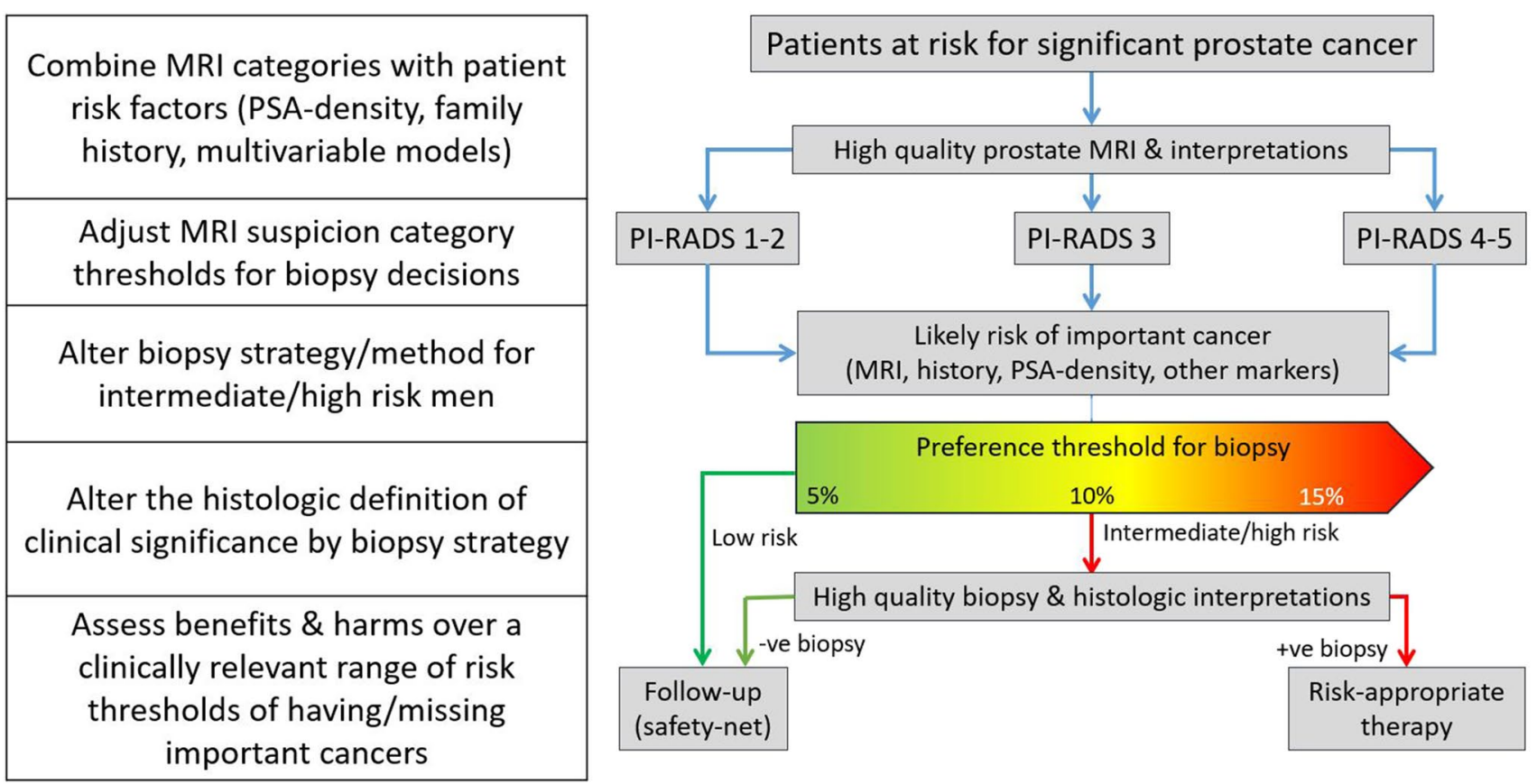

Fig. 1 Balancing the benefits and harms of MRI-directed biopsy results. Legend: There are multiple ways to optimize the balance of MRIdirected true and false results, weighing the benefits versus the harms. PI-RADS, Prostate Imaging-Reporting and Data System 
MRI-based strategies, including a clinical risk-based strategy where no biopsy was done in lower risk men with PI-RADS category 1-3 scores. Here, the clinical risk was assessed clinically by incorporating PSA-density values, DRE findings and family history with the MRI results. The reference standard was systematic biopsy for all patients plus targeted biopsies for MRI-identified lesions. They found that systematic and targeted biopsies only in men with positive MRI (MRI-focused) and the risk-based pathways showed the highest detection of International Society of Urological Pathology grade group $(\mathrm{GG}) \geq 2$ cancers. Moreover, the risk-based pathway was associated with a higher number of biopsies avoided. They noted that any MRI-directed pathways performed better than the systematic biopsies for the detection of $\mathrm{GG} \geq 2$ cancers while reducing the detection of $\mathrm{GG}=1$ tumours.

Based on the decision curve analysis, the net benefit of the risk-based pathway outperformed the other pathways within the typical range of "cancer averse" and "biopsy averse" clinical decision scenarios (5-30\%). These results are in line with the analysis of Deniffel et al. who also showed reductions in unnecessary biopsies in men with positive MRI by incorporating PSA-density values in canceraverse scenarios, and additionally the potential superiority of PI-RADS + PSA density compared to other non-calibrated logistic regression models [10]. The current study validates the extrapolations of Schoots et al. [14], on the likely benefit and harms of different MRI-based pathways. However, the current study results are not practice-changing for several reasons: the retrospective design of the study, the fact that the patients were highly selected (overall prevalence of cancer of 76\%) and were only biopsy-naïve, and the fact that all MRIs and biopsies were performed by the same operator. Additional retrospective and prospective studies are necessary to assess the consistency of the observations over a wider range of disease prevalence, populations and with radiologists and biopsy operators of varying experience. Only then can the risk-based MRI pathway be adopted as the default 'best practice' method of diagnosing men with suspected clinically important prostate cancers.

Funding The authors state that this work has not received any funding.

\section{Declarations}

Guarantor The scientific guarantor of this publication is Prof. Anwar R Padhani.

Conflict of interest The authors declare no competing interests.

Statistics and biometry No complex statistical methods were necessary for this paper.
Informed consent Written informed consent was not required for this study because this is an invited editorial and not the subject of a research article.

Ethical approval Institutional Review Board approval was not required because this is an editorial piece and not subject to a research article.

Study subjects or cohorts overlap Not relevant.

Methodology

- Editorial comment

\section{References}

1. Sathianathen NJ, Omer A, Harriss E et al (2020) Negative predictive value of multiparametric magnetic resonance imaging in the detection of clinically significant prostate cancer in the prostate imaging reporting and data system era: a systematic review and meta-analysis. Eur Urol 78:402-414. https://doi.org/10.1016/j.eururo.2020.03.048

2. Stonier T, Simson N, Shah T, et al (2020) The "Is mpMRI Enough" or IMRIE Study: a multicentre evaluation of prebiopsy multiparametric magnetic resonance imaging compared with biopsy. Eur Urol Focus 1-9. https://doi.org/10.1016/j.euf.2020.09.012

3. de Rooij M, Israël B, Barrett T et al (2020) Focus on the quality of prostate multiparametric magnetic resonance imaging: synopsis of the ESUR/ESUI recommendations on quality assessment and interpretation of images and radiologists' training. Eur Urol 78:483-485. https://doi.org/10.1016/j.eururo.2020.06.023

4. Moldovan PC, Van den Broeck T, Sylvester R et al (2017) What is the negative predictive value of multiparametric magnetic resonance imaging in excluding prostate cancer at biopsy? A systematic review and meta-analysis from the European Association of Urology Prostate Cancer Guidelines Panel. Eur Urol 72:250-266. https://doi.org/10.1016/j.eururo.2017.02.026

5. Mazzone E, Stabile A, Pellegrino F et al (2021) Positive predictive value of Prostate Imaging Reporting and Data System version 2 for the detection of clinically significant prostate cancer: a systematic review and meta-analysis. Eur Urol Oncol 4:697-713. https://doi. org/10.1016/j.euo.2020.12.004

6. Padhani AR, Barentsz JO, Weinreb J et al (2020) Variability of the positive predictive value of PI-RADS for prostate MRI across 26 centers: experience of the society of abdominal radiology prostate cancer disease-focused panel. Radiology 296:76-84. https://doi.org/10.1148/radiol.2020190646

7. Turkbey B, Rosenkrantz ABAB, Haider MAMA et al (2019) Prostate Imaging Reporting and Data System version 2.1: 2019 Update of Prostate Imaging Reporting and Data System version 2. Eur Urol 76:340-351. https://doi.org/10.1016/j.eururo.2019.02.033

8. Ahdoot M, Wilbur AR, Reese SE et al (2020) MRI-Targeted, Systematic, and Combined Biopsy for Prostate Cancer Diagnosis. N Engl J Med 382:917-928. https://doi.org/10.1056/NEJMoa1910038

9. Schoots IG, Padhani AR (2021) Risk-adapted biopsy decision based on prostate magnetic resonance imaging and prostate-specific antigen density for enhanced biopsy avoidance in first prostate cancer diagnostic evaluation. BJU Int 127:175-178. https://doi.org/10.1111/bju.15277

10. Deniffel D, Healy GM, Dong X et al (2021) Avoiding unnecessary biopsy: MRI-based risk models versus a PI-RADS and PSA density strategy for clinically significant prostate cancer. Radiology 300:369-379. https://doi.org/10.1148/radiol.2021204112

11. Bittencourt LK (2022) Risk-based MRI-directed diagnostic pathway outperforms non risk-based pathways in suspected prostate cancer biopsy-naïve men: a large cohort validation study. Eur Radiol. https://doi.org/10.1007/s00330-021-08407-6

12. Iczkowski KA, van Leenders GJLH, van der Kwast TH (2021) The 2019 International Society of Urological Pathology (ISUP) 
Consensus Conference on Grading of Prostatic Carcinoma. Am J Surg Pathol 45:1007. https://doi.org/10.1097/PAS.0000000000 001678

13. Van Calster B, Wynants L, Verbeek JFMM et al (2018) Reporting and interpreting decision curve analysis: a guide for investigators. Eur Urol 74:796-804. https://doi.org/10.1016/j.eururo.2018.08. 038
14. Schoots IG, Padhani AR, Rouvière O et al (2020) Analysis of magnetic resonance imaging-directed biopsy strategies for changing the paradigm of prostate cancer diagnosis. Eur Urol Oncol 3:32-41. https://doi.org/10.1016/j.euo.2019.10.001

Publisher's Note Springer Nature remains neutral with regard to jurisdictional claims in published maps and institutional affiliations. 